\title{
Review: cognitive behavioural therapy is effective for anger problems
}

Beck R, Fernandez E. Cognitive-behavioral therapy in the treatment of anger: a meta-analysis. Cognitive Therapy and Research 1998 Feb;22:63-74.

\section{Question}

In people with anger problems, does cognitive behavioural therapy $(\mathrm{CBT})$ reduce anger?

\section{Data sources}

Studies were identified using PsycINFO and Dissertation Abstracts (1970-95) with the terms anger control, anger treatment, and anger management.

\section{Study selection}

Studies were selected if they included $\geqslant 4$ patients, involved CBT for anger, and reported $\geqslant 1$ anger related dependent variable. Studies that used cognitive or behavioural interventions alone or that aimed only to promote relaxation were excluded.

\section{Data extraction}

Data were extracted on patient characteristics and numbers, study design, and dependent variables.

\section{Main results}

50 studies (1640 patients) were included. Patient groups studied included abusive parents and abusive spouses, schoolchildren, clinical adolescents, juvenile delinquents, inmates, and college students. Effect sizes were calculated from self reports of anger, dependent measures of anger and aggression, and behavioural ratings of aggression, and averaged across variables to produce 1 effect size per study. The effect sizes for the 50 studies ranged from -0.32 to 1.57 (SD 0.43) with all effect sizes but one positive in value. The grand mean weighted effect size was 0.70 which differed significantly from zero $(\mathrm{p}<0.001)$ and means that the average patient who received CBT fared better than $76 \%$ of patients who did not receive CBT. This corresponds to a treatment success rate of $67 \%$ for CBT compared with 33\% for patients who received control treatments.

\section{Conclusion}

Cognitive behavioural therapy is effective in patients with anger problems.

Source of funding: not stated.

For correspondence: Dr E Fernandez, Department of Psychology, Southern Methodist University, Dallas, TX 75275-0442, USA.Fax +1 214768 3910. (email efernand@mail.smu.edu).

\section{Commentary}

The experience of anger is part of everyday living. We consider anger a problem especially when it emerges with verbal or physical aggression, or both. Clinical knowledge has linked anger to health problems (eg, essential hypertension), psychosocial problems (eg, abusive behaviour), and some clinicians would like anger to be considered a disorder similar to anxiety and depression. In the foreward of a recent book on anger disorders, Albert Ellis states "Anger disorders, however, are common and require clear definition, specific diagnosis and effective treatment." Beck and Fernandez's metaanalysis shows that CBT is an effective treatment for the reduction of anger. However, support for CBT in a clinical population is still limited and the issues of clear definition and specific diagnosis of anger disorders remain elusive.

Practising clinicians who use CBT approaches will appreciate Beck and Fernandez's meta-analysis which complements the wealth of clinical and theoretical information on CBT currently available. Other clinicians may be encouraged by the empirical data to incorporate CBT into their practice. Some clinicians, however, may point to the need for more diversity in the population encompassed by the studies in the meta-analysis. Although CBT is viewed as the state-of-theart treatment for depression and anxiety disorders, it has lagged behind in its applications to anger control. ${ }^{2}$

The review by Beck and Fernandez correctly concludes that "ecological validity" for the management of anger for the clinical population still needs to be estab- lished. Most of the 50 studies included in the meta-analysis drew participants from educational and clinical settings. However, only 1 of the studies applied anger management to a "clinical adult" sample, 1 study used mentally retarded adults, 2 studies used forensic inpatients, and 7 of the 50 studies involved clinical adolescents. Studies treating oppositional children and adolescents are well represented, with half of the studies in the meta-analysis including them.

\section{Anthony Bellissimo, $\mathrm{MSc}, \mathrm{PhD}$ McMaster University \\ Hamilton, Ontario, Canada}

1 Kassinove H, editor. Anger disorders: definition, diagnosis, and treatment. Washington, DC: Taylor and Francis, 1995:xii-xiii.

2 Salkovskis PM, editor. Frontiers of cognitive therapy. New York: Guildford Press, 1996. 